\title{
Back-Projection Filtration Inversion of Discrete Projections
}

\author{
Imants Svalbe ${ }^{1}$, Andrew Kingston ${ }^{2}$, Nicolas Normand ${ }^{3}$, \\ and Henri Der Sarkissian ${ }^{3,4}$ \\ 1 School of Physics, Monash University, Melbourne, Australia \\ imants.svalbe@monash.edu \\ 2 Research School of Physical Sciences, Australian National University, \\ Canberra, Australia \\ andrew.kingston@anu.edu.au \\ 3 LUNAM Université, Université de Nantes, IRCCyN UMR CNRS 6597, \\ Nantes, France \\ \{nicolas.normand, henri.dersarkissian\}@univ-nantes.fr \\ ${ }^{4}$ Keosys, Saint-Herblain, France
}

\begin{abstract}
We present a new, robust discrete back-projection filtration algorithm to reconstruct digital images from close-to-minimal sets of arbitrarily oriented discrete projected views. The discrete projections are in the Mojette format, with either Dirac or Haar pixel sampling. The strong aliasing in the raw image reconstructed by direct back-projection is corrected via a de-convolution using the Fourier transform of the discrete point-spread function (PSF) that was used for the forward projection. The de-convolution is regularised by applying an image-sized digital weighting function to the raw PSF. These weights are obtained from the set of back-projected points that partially tile the image area to be reconstructed. This algorithm produces high quality reconstructions at and even below the Katz sufficiency limit, which defines a minimal criterion for projection sets that permit a unique discrete reconstruction for noisefree data. As the number of input discrete projected views increases, the PSF more fully tiles the discrete region to be reconstructed, the deconvolution and its weighting mask become progressively less important. This algorithm then merges asymptotically with the perfect reconstruction method found by Servières et al in 2004. However the Servières approach, for which the PSF must exactly tile the full area of the reconstructed image, requires $O\left(N^{2}\right)$ uniformly distributed projection angles to reconstruct $N \times N$ data. The independence of each (back-) projected view makes our algorithm robust to random, symmetrically distributed noise. We present, as results, images reconstructed from sets of $O(N)$ projected view angles that are either uniformly distributed, randomly selected, or clustered about orthogonal axes.
\end{abstract}

Keywords: Discrete tomography, image reconstruction from discrete projections, inverse problems. 


\section{Introduction}

A Mojette projection of a 2D digital image $\mathcal{I}$ is comprised of the sums of image pixel intensities that are located along parallel lines, oriented at some set of angles defined by pairs of co-prime integers, $\left(p_{i}, q_{i}\right)$ 3. A set of Mojette projections can be used to reconstruct, either approximately or exactly, an image of the original data. A discrete point-spread function (PSF) is defined by back-projection of a single point by a set of projected views, $\left(p_{i}, q_{i}\right)$. The PSF links the original image data to the Dirac Mojette back-projected image, $\mathcal{M}_{p q}$, through:

$$
\mathcal{M}_{p q}=\mathcal{I} * P S F_{p q}
$$

where $*$ denotes spatial convolution.

The Mojette Transform (MT) [3], is one of several inherently discrete image projection techniques, like the (closely related) Finite Radon Transform (FRT) 9, where each image projection is defined explicitly by the discrete image data. We prefer to approach image reconstruction by first defining the discrete image that we want to display and then deciding what sets of projected views are sufficient to reconstruct that image.

Our aim is to do tomography this way, i.e. to transform real, noisy projection data into a form that is discretely Mojette-like as possible, and then use the Mojette inverse to reconstruct the image.

Inversion from Mojette projection sets may also shine some theoretical light on the Katz Criterion [6] used in discrete tomography. Katz showed that any $N \times N$ image can be reconstructed exactly from a set of discrete projections $\left(p_{i}, q_{i}\right)$ provided $\max \left(\sum\left|p_{i}\right|, \sum\left|q_{i}\right|\right) \geq N$. Here we set $K=\frac{\max \left(\sum\left|p_{i}\right|, \sum\left|q_{i}\right|\right)}{N}$, so that $K=1$ for a projection set at the Katz limit, whilst a set with $K<1$ is below the Katz limit and cannot reconstruct an exact image. Katz specifies the spatial resolution and view angle requirements that permit exact digital inversion, but says nothing about approximate reconstructions, the effect of noise, the equivalence (or not) of different sets of angles, nor about the constraints imposed by the dynamic range of quantised image values.

There are other algorithms to invert Mojette projections, but these methods have severe limitations. The corner-based algorithm of Normand, and related geometric techniques [10, work only for noise-free projection sets that satisfy or exceed the Katz condition. Alternatively Mojette data can be mapped to the periodic form of the FRT, for which inversion by back-projection is exact, or else by applying the central slice theorem using Fourier [9] or number-theoretic transforms [12]. Direct inversion of the projection matrix is possible, but requires inverting very large matrices that are often ill-posed. Other methods, such as conjugate gradient [1] or partially ordered sets, require an iterative or statistical approach [4] that negate the advantages of using a direct reconstruction algorithm.

The intensity at each forward-projected Mojette bin is back-projected across the image reconstruction space, along the same discrete lines along which that 


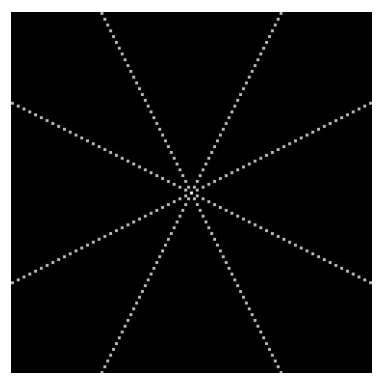

(a)

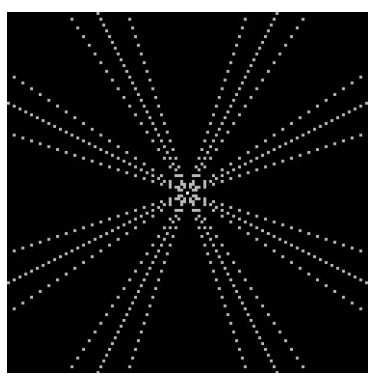

(b)

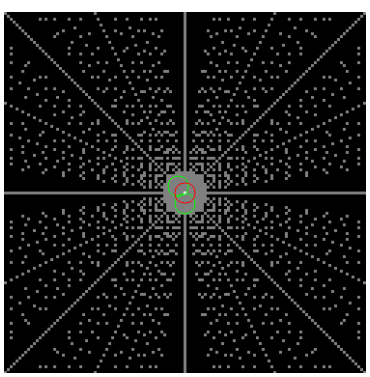

(c)

Fig. 1. Left to right: back-projected reconstruction of a single point using (a) 4, (b) 12 and (c) 60 discretely projected Dirac Mojette views. Image data confined to the area inside the red circle in (c) can be reconstructed exactly because the translated PSFs (green circles) intersect back-projected pixels that all have zero intensity.

data was projected (but with the mean projected sum, not the individual pixel values that make up each sum). The back-projection method is "blind" to other values in the projection data, it does not try to uncouple projection bins and pixel values. A back-projection algorithm is then tolerant of noise on the projections; it uses the same method for whatever data lies in each projection bin.

\section{Image Reconstruction and the Discrete PSF}

Fig. 1a shows four back-projected rays (at angles $( \pm 1,2),( \pm 2,1)$ ) for a single point located at the centre of an image. Back-projecting intensity values of 1.0 at the peak and $-1 /(4-1)$ elsewhere, results in a normalised intensity of 1.0 at their intersection point, zero along the projected rays and -0.333 elsewhere. Back-projecting 12 symmetric projections $((1,2),(1,3)$ and $(2,3)$ in Fig. 1b) with intensity 1.0 or $-1 /(12-1)$ gives 1.0 at the intersection point, zero along each of the 12 projected rays, and -0.0909 elsewhere. Fig. 1c shows the normalised Mojette back-projection for 60 projected views (the symmetric set of shortest vectors $(p, q)=(0,1)$ to $( \pm 3,7))$, yielding a centre value of 1.0 (white), the central circular region has value zero (grey), other pixels have value $-1 / 59$.

Note how the zero pixels increasingly tile the region that surrounds the intersection point. All translations of this PSF inside the red circle in Fig. 1c can be reconstructed exactly (as a single 1 on a background of 0 ). A PSF can only be translated as far as the green circles in that figure, before the negative background value (here the black pixels) will cause reconstruction errors. For a $59 \times 59$ image, 3208 Mojette projections $((0,1)$ to $(31,49))$ are needed to uniformly tile a disc of radius $R=58\left(\right.$ since $31^{2}+49^{2}=3362$ and $58^{2}=3364$ ). Hence direct, unfiltered, back-projection of discrete Mojette data works exactly, but it requires approximately $\left(\frac{6}{\pi}\right)^{2} 2 N(2 N-1)$ views to reconstruct an $N \times N$ image [13. This result confirmed earlier work done by Servières [12]. The shape 
of the image region of interest (ROI) also matters, as it determines how many, and which, array pixels need to be exactly tiled by the discrete PSF.

If you have only $M \ll N^{2}$ views, you can try to interpolate the missing $N^{2}-M$ views that you don't know, using the $M$ views that you do know 1312. For example, we can synthesize the projected view for $(13,14)$ from the known projection for $(1,1)$. Interpolation of digital profiles is tough work, especially for Dirac rather than the smoother Haar or higher-order spline projections.

If the image array is assumed to be periodic, the continuation of each projected ray (other than for $(1,0)$ and $(0,1)$ ) will pass across the ROI several times, increasing the number of pixels that are filled by back-projection.

For the FRT 9, where the array size is prime, $p \times p$, and for composite $N \times N$ arrays $7[8$, periodic back-projection can fully tile the ROI, the PSF is then "perfect", making exact inversion possible with $O(N)$ projections from an $N \times N$ image. A reconstruction method, for uniformly-distributed angle sets, that merges elements of the discrete Mojette, Fourier and compressed sensing techniques appeared recently [5].

\subsection{Reconstruction of Images Using a Finite, Discrete PSF}

Consider a finite PSF, such as our previous example composed of 60 views, which reconstructs, perfectly, any image inside the red circle of Fig. 2a, If we try to use this PSF to reconstruct a larger circular ROI (e.g. $60 \times 60$, the blue circle in Fig. 2a), we need to correct for each of the discrete un-corrected negative contributions that fall outside the flat zone of the blue PSF, i.e. all of the black points that lie inside the green circle shown in Fig. 2a,

A discrete, back-projected image, $\mathcal{M}_{p q}$, reconstructed from a set of view angles, $(p, q)$, is exactly equivalent to convolution of the discrete PSF with the original image, $\mathcal{I}$. This follows from the definition of discrete back-projection for Dirac Mojette data [3. This is easy to demonstrate; Fig. 3 shows an original image, $\mathcal{I}$, and the direct back-projected image, $\mathcal{M}_{p q}$, for the set of $60(p, q)$ angles shown in Fig. 2, The back-projected image is identical to the convolution of the original image data by the PSF shown in Fig. 2 (the differences on subtraction are $O\left(10^{-15}\right)$ and result from finite float computational precision). Then,

$$
\begin{gathered}
\mathcal{M}_{p q} * \operatorname{PSF}_{p q}^{-1}=\mathcal{I} \\
\mathcal{I}=F^{-1}\left\{\frac{F\left\{\mathcal{M}_{p q}\right\}}{F\left\{\mathrm{PSF}_{p q}\right\}}\right\}
\end{gathered}
$$

where $F\{\cdot\}$ denotes the 2D finite Fourier transform (FFT) of the zero-padded image data. Equation (3) is the basis for the de-convolution process presented in this work. Recovery of $\mathcal{I}$ from the direct, back-projected image $\mathcal{M}$, is contingent on the FFT of the PSF being well-conditioned or regularised. To ensure these conditions, a weighted version of the raw $\mathrm{PSF}$, denoted as $\mathrm{PSF}^{+}$in equation (6), is then used in (3). 


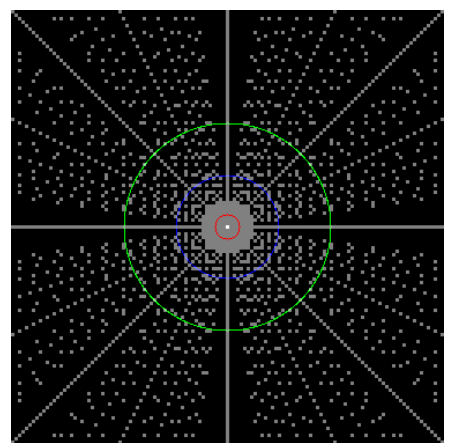

(a)

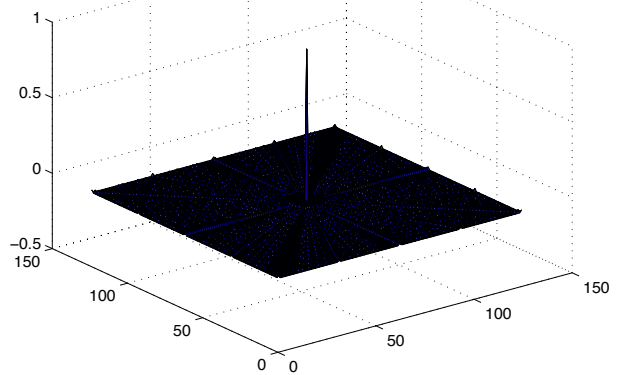

(b)

Fig. 2. Left: (a) Normalised, back-projected PSF reconstructed from 60 discrete projections at angles $(p, q)$ using the shortest vector lengths (white $=1$, grey $=0$, black $<0$ ). Images confined inside the red circle (with a diameter of about 23 pixels) can be reconstructed exactly by direct back-projection. Reconstruction of images lying inside the blue circle, with diameter 60 pixels, using the same 60 projected views, requires correction for all of the missing (black) back-projected points the lie inside the green circle with diameter 120 pixels. Right: (b) a 3D view of the PSF in (a).

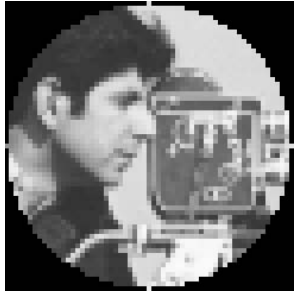

(a)

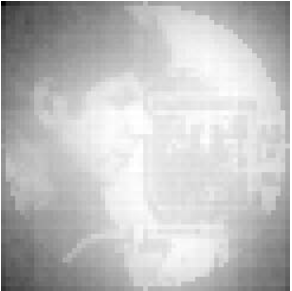

(b)

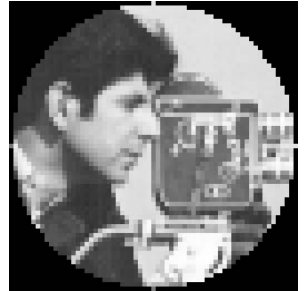

(c)

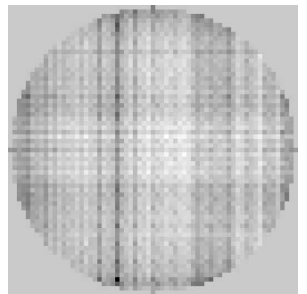

(d)

Fig. 3. (a): Original image $\mathcal{I}$, circular ROI diameter 60 pixels. (b): Raw back-projected reconstruction from 60 discrete Mojette views of the original data. Image (b) is exactly the same as the result obtained by convolving image (a) with the discrete PSF shown in Fig. 2b (c): Reconstructed image from the 416 Mojette shortest $(p, q)$ views $(65 \times 65$ pixels, $K=57 \gg 1$ ), PSNR $=46.62$, MSE $=1.30$. This is about $10 \%$ of the number of views required for exact, unfiltered Mojette back-projection. (d): reconstruction errors for this image are structural and arise from inversion of the PSF; they are not evidently strongly image-related.

\subsection{Image Reconstruction Examples with $K \gg 1$}

We verify this approach by applying (3) to reconstruct images from discrete projection sets comprised of many views, but far fewer than the $O\left(N^{2}\right)$ views required for exact $N \times N$ reconstruction. Fig. 3c shows an example image, reconstructed from 416 Mojette projections, that were obtained from a circular ROI in $65 \times 65$ discrete image data (a cropped portion of the "cameraman" image). 
Adding normally distributed noise to each of the 416 projections only slightly reduced the reconstructed image PSNR (from 46.62 to $43.8 \pm 0.2$ ), confirming the robustness of the back-projection approach. The reconstructed images for $K \gg 1$ are of high quality, but our aim here is to reduce the number of discrete projections required, so that $K \simeq 1$, or preferably, $K<1$. Using fewer projection directions makes the outer zones of the PSF increasingly sparse and the flat zone of zeros smaller, so that the PSF inverse becomes less well-conditioned and requires some regularisation.

\section{Regularisation of the PSF}

Our approach to recover images for projection sets where $K \simeq 1$, is to weight the image of the PSF, in the form as shown in Fig. 2, so that the outer regions of the PSF are made smoother and closer to zero, whilst preserving the central flat zone. These weights should be smooth and close to unity for $O\left(N^{2}\right)$ projected views, where no or little correction of the back-projected image is required. For increasingly sparse views, the weights should be closer to zero around the periphery of the PSF, and remain strongly discretised along those directions where more correction is needed.

For sets of projected views that exceed the Katz Criterion $(K \geq 1)$, we construct a weight function, that we call $W_{p n}$, that reflects the correlation between the spatial distribution of points across the region of support in the PSF that are correctly back-projected and the distribution of points where the back-projected data is absent.

We generate first an image $(p)$ of the PSF that contains the correctly backprojected points over the region of image support. These points are set to one, all other points are set to zero. We generate a second image $(n)$ of the PSF that contains the (complementary) set of uncorrected back-projected points over the region of image support. These points are set to one, all other points are set to zero. We then cross-correlate these distributions, across the full extent of the back-projected space, as

$$
W_{p n}=(p \star n) *(D \star D)
$$

where $\star$ denotes the cross-correlation product and $D$ has the uniform value of 1 over the image region of support and is otherwise zero. Pixels of $W_{p n}$ that fall within the central flat zone of the PSF (the area of perfect reconstruction) should not be down-weighted, so the weight for these pixels is set to 1 . The function $W_{p n}$ is then normalised to have maximum value 1, as shown in Fig. 4a.

For sets of projected views that fall below the Katz Criterion $(K \leq 1)$, the back-projected area becomes increasingly sparse outside the small flat central zone of exact reconstruction and the region of support, $D$, plays an increasingly smaller contribution. For these cases, the weight function, that we call $T_{p n}$, is constructed as a direct correlation of the spatial distributions of the correctly back-projected points in the PSF vs the non-back-projected points;

$$
T_{p n}=p \star n
$$




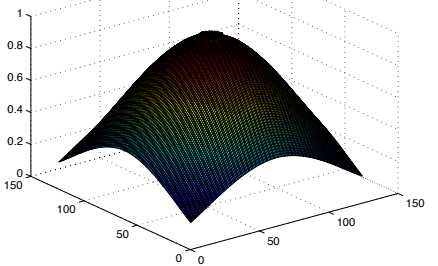

(a)

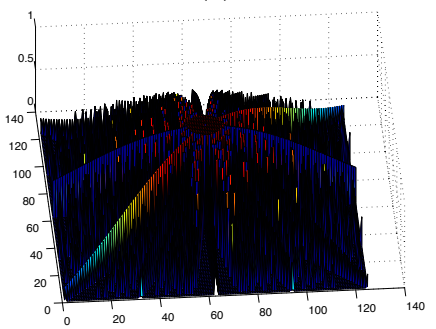

(c)

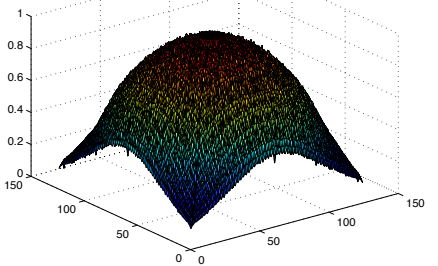

(b)

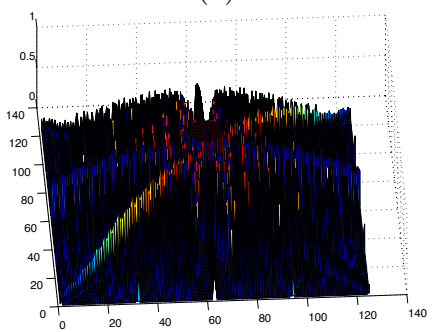

(d)

Fig. 4. Top row: (a-b) Left: (a) Weight function $W_{p n}$ for the PSF for the set of 52 angles $((1,0)$ to $( \pm 2,7)), 63 \times 63$ image, circular region of support. Right: (b) Weight function $T_{p n}$ for the same PSF as in (a).

Bottom row: (c-d) Left: (c) The PSF weighted by $W_{p n}$ for $K>1$. Right: (d) the PSF weighted by $T_{p n}$ for $K<1$. The central spike of the PSF has been suppressed here to enhance the display of the small values at the periphery.

The weights for those pixels that lie within the central flat zone of the PSF are set to 1 , with the net result normalised, as shown in Fig. 4b. Weights $W_{p n}$ and $T_{p n}$ multiply, point to point, the raw PSF image values,

$$
\mathrm{PSF}^{+}= \begin{cases}\mathrm{PSF} \odot W_{p n}, & \text { if } K>1 \\ \mathrm{PSF} \odot T_{p n}, & \text { if } K<1\end{cases}
$$

where $\odot$ denotes the element-wise multiplication of $2 \mathrm{D}$ vectors.

\section{Reconstruction Results for De-convolution of Back-Projected Images Using the Weighted PSF}

We applied the weightings $W_{p n}$ and $T_{p n}$ to the PSF (as shown in Fig. 4c d) and recorded the PSNR for images that were reconstructed, using equations (3) and (6), for varying numbers of projected views. Here all angle sets are comprised of the shortest $(p, q)$ vectors. The results are given in Table 1 for image sizes of $63 \times 63$ and $127 \times 127$, respectively. At the Katz point, $K=1$, the PSNR values for reconstruction from projections after adding normally distributed noise have 
Table 1. PSNR values for two images of sizes of $63 \times 63$ and $127 \times 127$, reconstructed using a weighted PSF, as a function of the number of projected views (shortest $(p, q)$ vector angle sets)

\begin{tabular}{cllll}
\hline Image size $K$ (Katz' value) & Projections count Weight $W_{p n}$ & Weight $T_{p n}$ \\
\hline 0.59 & 20 & 18.89 & 18.67 \\
0.81 & 24 & 19.98 & 19.93 \\
$63 \times 63$ & 1 & 28 & 21.63 & 21.63 \\
& 1.22 & 32 & 22.92 & 22.73 \\
& 2.52 & 52 & 27.61 & 26.76 \\
3.67 & 64 & 30.08 & 28.54 \\
6.46 & 96 & 34.34 & 31.06 \\
9.89 & 128 & 35.74 & 31.62 \\
\hline 0.5 & 28 & 17.77 & 17.78 \\
0.61 & 32 & 18.90 & 18.75 \\
0.73 & 36 & 19.30 & 19.38 \\
0.84 & 40 & 20.30 & 20.09 \\
$127 \times 127$ & 0.98 & 44 & 21.35 & 20.92 \\
1.11 & 48 & 22.54 & 21.66 \\
3.2 & 96 & 29.70 & 26.95 \\
4.91 & 128 & 32.74 & 28.55 \\
9.11 & 192 & 35.01 & 29.44 \\
\hline
\end{tabular}

also been included. Those results provide further confirmation of the robustness of our direct inversion method.

We observed that weight $W_{p n}$ performs better for $K>1$, whilst the reconstruction results for weight $T_{p n}$ become slightly better than for weight $W_{p n}$ for $K<1$, especially in larger images. For example, a $509 \times 509$ portion of the Lena image, reconstructed using the 96 shortest angles, where $K=0.8$, yields a PSNR of 19.80 for $T_{p n}$ and 18.41 for $W_{p n}$.

The only other tool used to regularise the inverse of the PSF is to apply a threshold test to the Fourier coefficient values before taking their inverse. If the Fourier coefficient of the (weighted) PSF at $(u, v)$ was less than a selected fixed value, that coefficient was set to the mean of all above-threshold $3 \times 3$ neighbouring coefficients. The choice of this threshold value turned out to be relatively insensitive; optimising its value made relatively small differences to the final PSNR values. When reconstructing any images, we keep track of the number of times the threshold is reached, to indicate if the threshold needs to be modified. For example, at $K=0.59$, for 20 angles, PSNR $=18.89$ improves to 19.45 after scanning across a range of threshold values to optimise the PSNR.

The size of the zero-padded region can be adjusted to be made (symmetrically) slightly smaller or larger. This can change the reconstructed PSNR (again, only slightly), as it may affect the degree of aliasing by the discretisation of the finite Fourier transform at specific frequencies. 


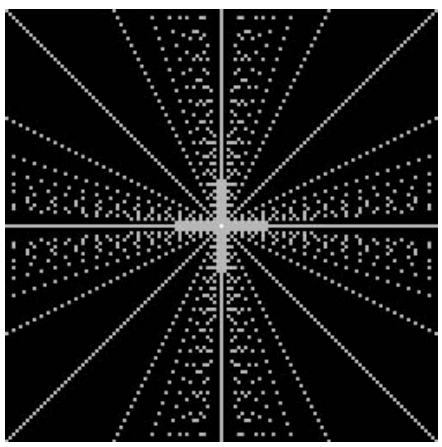

(a)

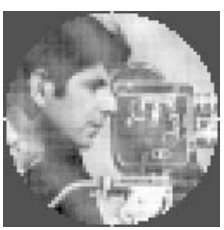

(b)

Fig. 5. Reconstruction of an image from a clustered set of 52 projection angles $( \pm 1, i),( \pm i, 1) ; 0 \leq i \leq 13\}$, for which $K=3.29$. Left: (a) the raw clustered view PSF $(125 \times 125)$. Right: $(\mathrm{b})$ the reconstructed image $\left(63 \times 63\right.$, using weight $\left.T_{p n}\right)$, with $\mathrm{PSNR}=23.33$. After adding normally distributed noise, $\mathrm{PSNR}=23.1 \pm 0.1$.

\section{Reconstruction Using Different Distributions of Projected View Angles}

\subsection{Clustered Projection Angles}

We reconstructed images from sets of projected views that are clustered around $0^{\circ}$ or around $90^{\circ}$. We do this by selecting discrete angles $(p, q)$ corresponding to $( \pm 1, i)$ and $( \pm i, 1)$, for integers $0 \leq i \leq n$. To maintain four-fold angle symmetry, we usually increment the number of angles in steps of 4 . The strongly nonuniform distribution of angles makes the shape of the PSF less uniform and thus more difficult to correct. Here the discrete weight $T_{p n}(\mathrm{PSNR}=23.33)$ performs better than weight $W_{p n}(\mathrm{PSNR}=20.08)$, even for $K>1$. An example reconstructed image is shown in Fig. 5 .

\subsection{Randomly Distributed Projection Angles}

We generated a random set of $M$ projection angles selected from a range of $(p, q)$ vectors that was three times larger than for the shortest angle set for $M$ projections. As $p$ and $q$ can now be much larger than for the shortest set, the number of bins in these projections, as given by $n_{\text {bin }}=(|p|+|q|)(N-1)+1$, is also larger.

Whilst randomising the projection angles generally decreases the reconstructed image quality because the PSF is less uniform, the use of large $p$ and $q$ values generally improves the discrete reconstructions. The large $|p|+|q|$ discrete projections are less heavily summed, because there are more projection bins, but the projected ray passing into each bin intersects fewer pixels. Forcing the inclusion of the projections $(1,0),(0,1)$ and $( \pm 1,1)$ as part of the "random" set improves the results, as those projections carry significant information about the image. 


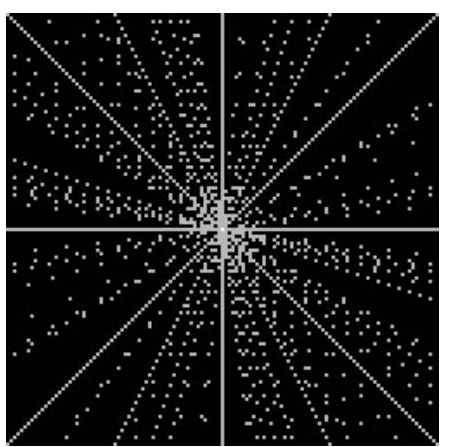

(a)

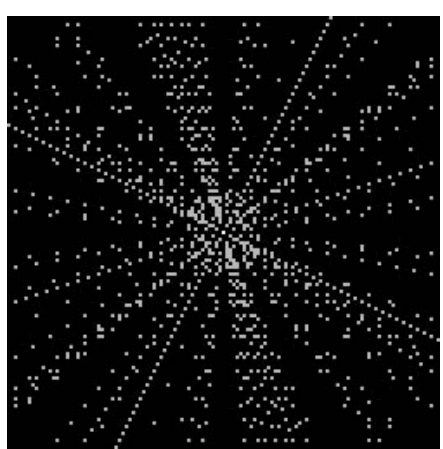

(b)

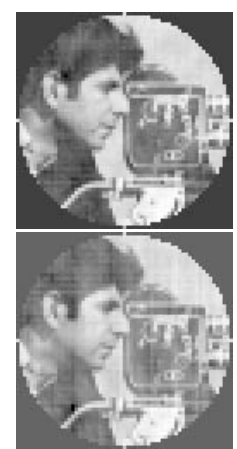

(c)

Fig. 6. (a-b): PSF $(125 \times 125)$ for two random sets of 52 projection angles. (a): Including $(1,0),(0,1)$ and $( \pm 1,1)$. (b): Excluding $(1,0),(0,1)$ and $( \pm 1,1)$. (c): Corresponding $63 \times 63$ reconstructed images using filter $W_{p n}$. Top image, PSNR $=31.31$, bottom image, PSNR $=26.33$. After adding normally distributed noise PSNR $=27 \pm 1$ and $24.5 \pm 1$ respectively.

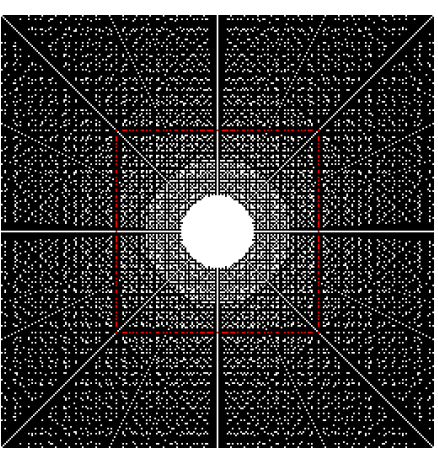

(a)

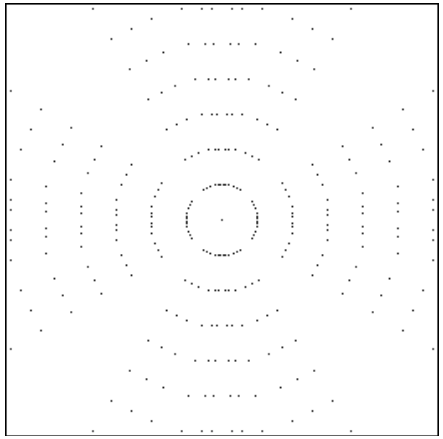

(b)

Fig. 7. (a): Raw discrete PSF $(129 \times 129)$ for the 440 shortest $(p, q)$ angles. The highlighted portion of the rows and columns 60 pixels away from the PSF centre are heavily tiled by back-projection. In contrast, pixels along the rows and columns a prime distance from the centre (e.g. 59 and 61) are sparsely tiled. (b): Differences in the discrete PSF for the projection sets comprised of the 440 and 416 shortest angles emphasize the bias towards better reconstruction along the horizontal and vertical directions.

The PSF weight $W_{p n}$ performs slightly better here (mean PSNR $=28.5 \pm 1.5$ ) than does weight $T_{p n}$ (mean PSNR $=25.5 \pm 1.5$ ), as the view angles are, on average, more uniform and $K>1$. Here $K=4.5 \pm 0.3$, see Fig. 6 .

\subsection{Discrete Image Reconstruction Errors}

Our reconstruction errors occur predominantly along the image rows, columns and diagonals (as in Fig. 3). Partly this effect may be due to the method we 
used to weight and invert the PSF, but another contribution comes from the non-random distribution of $(p, q)$ points as they get back-projected.The number of back-projected points and their inclusion in the PSF depends on the set of $(p, q)$ view angles, but also on the size of the image ROI to be reconstructed.

Fig. 7a shows a PSF back-projected from the 440 discrete shortest $(p, q)$ angles (as a $129 \times 129$ pixel image). Entries in the PSF that arise from projection $(p, q)$ are back-projected as points located $(n p, n q)$ pixels from the centre of the PSF. Many back-projected points will lie on columns or rows that are 60 pixels distant from the centre, as $n$ can be any of the many factors of 60 ; $n=\{2,3,4,5,6,10,12,15,20,30\}$.

However the PSF has few (almost zero) back-projected points along columns or rows located 59 and 61 pixels from the centre, as these numbers are prime. A $61 \times 61$ portion from the image in Fig. $3 \mathrm{c}$ reconstructs (using an unweighted PSF at 440 shortest views) with a PSNR of 41.76 , whereas a $59 \times 59$ portion of the same data reconstructs with $\mathrm{PSNR}=47.68$, using identical views. Including or excluding row and column 60 from the reconstructed ROI makes a large difference to the PSF that, in turn, affects the reconstructed PSNR.

Strong, local changes at the edges of the PSF may also explain why we obtain reconstructions for $65 \times 65$ images using 416 angles $(\operatorname{PSNR}=46.62$, last $(p, q)=$ $(12,17))$ that are, uncharacteristically, slightly better than those for a much larger angle set of 440 views $(\operatorname{PSNR}=46.41$, last $(p, q)=(4,21))$. For $63 \times 63$ images we obtain PSNR $=45.52$ for 416 views, PSNR $=46.69$ at 440 views, because part of the outer circle of back-projected points shown in Fig. 7b (arising from the $(4,21)$ view), are now excluded.

\section{Summary, Conclusions and Future Work}

We presented, in this work, an approach to reconstructions that uses a weighted version of the raw back-projected discrete PSF to recover, by direct deconvolution, the original digital image from its direct back-projected reconstruction. As this is a linear system, the same approach could equally be achieved by filtering the Mojette data in 1D before back-projection, as was originally suggested by Andrew Kingston. Correction of 3D back-projected images, via the inverse of the $3 \mathrm{D}$ PSF, is a natural extension of this $2 \mathrm{D}$ approach.

This filtered back-projection approach also tolerates the presence of significant levels of noise in the projected data. Computation of these filtered back-projected images is fast to compute, especially if the Fourier transform of the discrete PSF and the associated weight functions $\left(T_{p n}\right.$ or $\left.W_{p n}\right)$ are known.

The relatively high accuracy of our reconstructions and the rapidity with which they can be obtained would provide high quality initial-image estimates that may enhance the convergence rate for slower, statistical iterative reconstruction methods.

The use of an exact, algebraic approach to pre-filter each projection also seems possible. Nicolas Normand has shown that direct inversion is possible for projection data cast as a Vandermonde matrix and has also shown that 
Moore-Penrose pseudo-inverse techniques may be used to invert projection matrices (unpublished work). These new techniques complement the existing "rowsolving" techniques [12] or methods based on the central-slice theorem [9] that map between Mojette (or FRT) data and image space.

An enhanced ability to invert arbitrary sets of Mojette data will permit detailed studies to be undertaken to understand how the grey-level quantisation of a digital image affects image reconstruction, as seen via the Katz criterion. Our method has already been applied to reconstruct binary and ternary image data from sparse sets of discrete projections (unpublished work).

Acknowledgments. The IVC group hosted a visit by Imants Svalbe to work in their laboratory from November to December, 2013. Polytech Nantes provided financial support to him as a Visiting Invited Professor for that period.

\section{References}

1. Chandra, S., Svalbe, I.D.: Exact image representation via a number-theoretic Radon transform. IET Computer Vision (accepted November 2013)

2. Chandra, S., Svalbe, I.D., Guédon, J., Kingston, A.M., Normand, N.: Recovering missing slices of the discrete Fourier transform using ghosts. IEEE Trans. Image Process. 21(10), 4431-4441 (2012)

3. Guédon, J., Normand, N.: Direct Mojette Transform. In: Guédon, J. (ed.) The Mojette Transform: Theory and Applications, pp. 37-60. ISTE-WILEY (2009)

4. Herman, G.T., Kuba, A. (eds.): Advances in Discrete Tomography and its Applications. Birkhäuser, Boston (2007)

5. Hou, W., Zhang, C.: Parallel-Beam CT reconstruction based on Mojette transform and compressed sensing. Int. J. of Computer and Electrical Eng. 5(1), 83-87 (2013)

6. Katz, M.B.: Questions of uniqueness and resolution in reconstruction from projections. Springer, Berlin (1978)

7. Kingston, A.M., Svalbe, I.D.: Generalised finite Radon transform for $\mathrm{N} \times \mathrm{N}$ images. Image and Vision Computing 25(10), 1620-1630 (2007)

8. Lun, D., Hsung, T., Shen, T.: Orthogonal discrete periodic Radon transforms, Parts I \& II. Signal Processing 83(5), 941-971 (2003)

9. Matúš, F., Flusser, J.: Image representation via a finite Radon transform. IEEE Trans. Pattern Anal. Mach. Intell. 15(10), 996-1006 (1993)

10. Normand, N., Kingston, A., Évenou, P.: A Geometry Driven Reconstruction Algorithm for the Mojette Transform. In: Kuba, A., Nyúl, L.G., Palágyi, K. (eds.) DGCI 2006. LNCS, vol. 4245, pp. 122-133. Springer, Heidelberg (2006)

11. Servières, M., Idier, J., Normand, N., Guédon, J.: Conjugate gradient Mojette reconstruction. In: SPIE Medical Imaging, pp. 2067-2074. SPIE (2005)

12. Servières, M., Normand, N., Guédon, J.: Interpolation method for the Mojette transform. In: SPIE Medical Imaging, SPIE, San Diego, CA (2006)

13. Svalbe, I.D., Kingston, A.M., Guédon, J., Normand, N., Chandra, S.: Direct Inversion of Mojette Projections. In: Int. Conf. on Image Processing, Melbourne, Australia, pp. 1036-1040 (2013) 